\title{
Competitiveness of Young Football Players in the Japan Football Association Social Action Program
}

\author{
Hiroaki Matsuyama1 ${ }^{*}$, Takahiro Matsutake2, Hiroyuki Horino ${ }^{3}$, Hironobu Tsuchiya ${ }^{2}$ \\ ${ }^{1}$ Graduate School of Osaka University of Health and Sport Sciences, Osaka, Japan \\ ${ }^{2}$ Osaka University of Health and Sport Sciences, Osaka, Japan \\ ${ }^{3}$ Waseda University, Tokyo, Japan \\ Email: ${ }^{*}$ mhiroaki0831@yahoo.co.jp
}

Received 27 February 2015; accepted 13 April 2015; published 14 April 2015

Copyright (C) 2015 by authors and Scientific Research Publishing Inc.

This work is licensed under the Creative Commons Attribution International License (CC BY).

http://creativecommons.org/licenses/by/4.0/

c) (i) Open Access

\section{Abstract}

Conditions affecting the competitiveness of young group athletes in different countries were investigated through a comparative evaluation of opinions expressed by coaches and instructors dispatched overseas through the JFA Social Action Program in Asia. Results of a one-way analysis of variance on responses scores related to football teams in different countries, which was conducted after calculating group means and standard deviations indicated that physical strength and mental strength scores were significantly higher in teams of the high ranking group than in teams of low the ranking group classified according to FIFA rankings. These results suggest that there is an urgent need to establish football teams on a school wide basis and to become affiliated organizations of professional football clubs in countries in the low ranking group, in order to develop continuous training for each category of young athletes. Such training would be necessary to increase physical strength and decrease differences in physical strength between low and high ranking groups of countries. Similarly, it would be important for young athletes continue training, participate in games, and feel a sense of improvement and fulfillment in order to bridge the gap in mental strength scores between low and high ranking groups. A comprehensive balance between training, playing games, and a sense of fulfillment is expected to lead to prioritized training and to the development of a sense of pride among young athletes representing their country by playing football.

\section{Keywords}

Japan Football Association (JFA), Social Action Programs in Asia, Overseas Dispatched Instructors,

\footnotetext{
*Corresponding author.
}

How to cite this paper: Matsuyama, H., Matsutake, T., Horino, H., \& Tsuchiya, H. (2015). Competitiveness of Young Football Players in the Japan Football Association Social Action Program. Advances in Physical Education, 5, 94-102. 


\section{Football}

\section{Introduction}

Recently, there has been growing interest in global sports competitions. Correspondingly, the scales of these competitions are expanding as an increasing number of countries are participating in Olympic Games and the Federation Internationale de Football Association (FIFA) (2014) World Cup. The number of member nations of the International Olympic Committee (IOC) (2014) and FIFA greatly exceeds the number of United Nations member nations. This shows that sports is spreading worldwide and is gradually gaining acceptance as a global standard (Kobayashi, 2001).

The Japan Football Association (JFA) is a member of the IOC and the Japan Olympic Committee (JOC). In this increasingly globalized world, the JFA is engaged in exchanges of human resources to develop Asian football as a part of its Social Action Programs in Asia. The objective of this program is to promote exchanges between Japan and neighboring Asian countries and regions. As an effort to promote the coexistence and prosperity of countries in Asia, the JFA educates instructors to nurture teams, coaches, and referees, and provides physical and intellectual support programs (JFA, 2013a). The objective of the Social Action Programs in Asia is to "give children a bright future through football and contribute to the diffusion and development of Asian football." (JFA, online) The JFA promotes various programs as a model association to support members of the Asian Football Confederation (AFC) consisting of 47 member nations and regions. By March 2014, the JFA has dispatched 45 national coaches (including coaches of youth teams) and instructors to a total of 19 Asian countries for youth development (JFA, online).

The degree of football skills is not necessarily high in countries where coaches and instructors have been dispatched. According to the FIFA rankings, Zambia is presently ranked $62^{\text {nd }}$ in the FIFA ranking and is the highestranking country among those participating in JFA Social Action Programs in Asia (FIFA, online). Also, Japan is the highest ranking country among 47 members of the AFC. Moreover, Japan is presently ranked $53^{\text {rd }}$ among countries in the FIFA ranking, in which only nine Asian teams are ranked among the top 100. It is considered that the diffusion and development of football in Asian countries will improve the level of football skills in the Asian region and eventually improve their FIFA rankings. To this end, each country needs to have a clear policy regarding measures for improving football and endeavor to develop and promote Asian football to an international level. In this regard, it is critically important to improve the aptitude of local football instructors (JFA, 2010).

Previously dispatched official JFA licensed instructors to Asian countries have reported on the countries to which they were dispatched, as well as current trends and issues related to the appointed country in academic reports and papers (Reports by the JFA; Sone, 2008; Matsuyama et al., 2014). Such reports indicate that it is necessary to review coaching systems for young people and improve the aptitude of instructors, in order to increase the competitiveness of these countries. However, with the exception of reports about instructors, there have been no previous investigations of the actual conditions of young players in different countries. To date, mainly the FIFA rankings have been used to evaluate the level of each country. However, this ranking only evaluates the most representative teams, or "A" teams in each country and therefore, the ranking is unlikely to reflect the actual conditions of young players. We considered that it is necessary to grasp the reality of the conditions faced by young players in different countries, in order to develop programs for further growth and achieve coexistence and prosperity in Asia.

For these reasons, the present study was designed to elucidate the actual conditions of competitiveness in young players in different countries, based on activities of instructors dispatched overseas by the Social Action Program in Asia. Also, we intended to develop improved measures and training contents that would be vital for the development of football in these countries.

\section{Method}

Head coaches and coaches $(\mathrm{N}=45)$ participated in the JFA's Social Action Program in Asia. Of these participants, three national futsal coaches, two referees' instructors, and four head coaches and coaches of women's national football teams were excluded from the study. We conducted a questionnaire survey with the remaining 36 head coaches and coaches of men's national football teams, and youth teams. Those providing invalid res- 
ponses, with incomplete or wrong responses were excluded from the study. There were 24 valid respondents used in the analysis (a valid response rate of $66.7 \%$ ). The survey was conducted in line with research ethics by using either one of the following two methods. In the first method, researchers sent a questionnaire to respondents after obtaining their consent over the telephone and respondents returned the questionnaire by post. In the second method, researchers visited respondents who were head coaches, briefly explained the objective of the survey, and had them complete the questionnaire after obtaining their consent, and collected the questionnaires immediately after completion.

\subsection{Survey Contents}

We developed the questions based on four items reported by Matsuyama et al. (2014): technical aspects, tactical aspects, physical strength aspects, and mental strength aspects of the game. Then, we applied the content of the four items based on the results of a preliminary survey conducted by Abe et al. (2012). The contents of the four items were as follows. Technical aspects that included "Passing (shooting) techniques," "Trapping techniques," and "Dribbling, as well as techniques" for "keeping possession of the ball." Tactical aspects that included "Understanding tactics," "Situational awareness skills," "Positioning," and "Instructions.” Physical strength aspects that included "Endurance," "Speed," "Muscle strength," "Agility," and "Physique.” Mental strength aspects that included "Ambition," "Goal setting," "Competitiveness," "Motivation for playing," "Obedience," "Trust” and "Leadership."

Next, based on the four items developed by Matsuyama et al. (2014) and the contents of the fours items obtained from the preliminary survey conducted by Abe et al. (2012), as well as the Players' Competitiveness Evaluation Scale consisting of 42 items developed by Abe et al. (2012), we requested three head coaches of university football teams and eight graduate students with experience in football to select a total of 20 items as the criteria for evaluating players during international coaching. The final, 20-item questionnaire consisted of seven items assessing technical aspects, three items assessing tactical aspects, five items assessing physical strength aspects, and five items assessing mental strength aspects of the game.

The beginning of the questionnaire consisted of the following instructions: "For each question below, please circle the number that corresponds with the extent to which you think the item applies to players in the country." Respondents evaluated each item on a five-point Likert scale ranging from 5 (Strongly agree) to 1 (Strongly disagree). Each response was scored with a score between 1 - 5 points and the points were added to obtain the total score. We used the total score for comprehensive evaluation and as the competitiveness evaluation score.

\subsection{Survey Period}

The survey was conducted between April and August 2014.

\subsection{Survey Method}

We classified appointed countries into 3 groups based on the FIFA rankings announced immediately after instructors participating in the Social Action Program in Asia left for their appointed countries. The three groups consisted of the high ranking group $\left(\mathrm{n}=8,92^{\text {nd }}\right.$ position- $138^{\text {th }}$ position), the medium ranking group $\left(\mathrm{n}=8,141^{\text {st }}\right.$ position- $187^{\text {th }}$ position), and the low ranking group $\left(n=8,190^{\text {th }}\right.$ position-non-member nations). In Table 1 , we have listed the three groups that were selected on the basis of the FIFA rankings of the period immediately after the participating instructors in the Social Action Program in Asia left for their appointed countries. We conducted a comparative review between the high ranking group, the medium ranking group, and the low ranking group shown in Table 1.

\subsection{Statistical Analysis}

We conducted a one-way analysis of variance (ANOVA) on the measured values obtained in the present experiment by using IBM SPSS Statistics 21. Then, we conducted multiple comparisons by using the Bonferroni method for variables with significant differences. The significance level of statistical analysis was set at $5 \%$.

\section{Results}

We analyzed the mean and standard deviation and then conducted an ANOVA on the overall scores of the four 
Table 1. FIFA ranking of Asian countries (high/medium/low).

\begin{tabular}{|c|c|c|c|c|}
\hline & Index & Country & Period & FIFA Ranking \\
\hline 1 & \multirow{9}{*}{$\begin{array}{l}\text { High rank } \\
\text { group }\end{array}$} & Republic of Zambia & Mar-09 & 92 \\
\hline 2 & & Syria Arab republic & Sep-10 & 94 \\
\hline 3 & & Syria Arab republic & Jan-06 & 100 \\
\hline 4 & & Syria Arab republic & Jun-09 & 104 \\
\hline 5 & & Republic of Singapore & Apr-11 & 112 \\
\hline 6 & & Syria Arab republic & Jun-07 & 114 \\
\hline 7 & & Kyrgyz Republic & Jul-13 & 137 \\
\hline 8 & & People’s Republic of Bangladesh & Sep-11 & 138 \\
\hline 9 & & Republic of Tadjikistan & Jul-11 & 141 \\
\hline 10 & \multirow{7}{*}{$\begin{array}{l}\text { Medium rank } \\
\text { group }\end{array}$} & Democratic Socialist Republic of Sri Lanka & Apr-07 & 160 \\
\hline 11 & & Democratic Socialist Republic of Sri Lanka & Jun-09 & 161 \\
\hline 12 & & Kingdom of Cambodia & Dec-10 & 166 \\
\hline 13 & & Republic of China & May-12 & 168 \\
\hline 14 & & Republic of the Philippines & Mar-02 & 175 \\
\hline 15 & & Kingdom of Cambodia & Jun-09 & 179 \\
\hline 16 & & Kingdom of Cambodia & Dec-13 & 187 \\
\hline 17 & \multirow{8}{*}{$\begin{array}{l}\text { Low rank } \\
\text { group }\end{array}$} & Lao People’s Democratic Republic & Jul-12 & 190 \\
\hline 18 & & Republic of the Philippines & Aug-06 & 192 \\
\hline 19 & & Macao Special Administrative Region & Feb-06 & 192 \\
\hline 20 & & Kingdom f Bhutan & Apr-08 & 200 \\
\hline 21 & & Kingdom f Bhutan & May-12 & 201 \\
\hline 22 & & Territory of United states of America(Guam) & Feb-05 & 205 \\
\hline 23 & & Commonwealth of the Northern Maliana Island(Saipan) & Jul-11 & Non-member \\
\hline 24 & & Commonwealth of the Northern Maliana Island(Saipan) & Mar-10 & Non-member \\
\hline
\end{tabular}

items on the Players' Competitiveness Evaluation Scale. The results of this analysis are shown on Table 2. We also analyzed the mean and standard deviation and then conducted an ANOVA on each factor score of the 20 items of the Players' Competitiveness Evaluation Scale. Table 3 shows the results of this analysis. The results indicated a main effect of physical strength and mental strength scores. Post-hoc tests indicated that physical strength score was significantly higher in the high ranking group than in the low ranking group $(F(1,22)=4.09$, $p<0.05)$. Moreover, the mental strength score was significantly higher in the high ranking group than the low ranking group $(F(1,22)=4.25, p<0.05)$.

Additionally, results of each factor score indicated that the score of "Have the power to keep the ball” related to technical aspects were significantly higher among the high ranking group compared to the low ranking group $(F(1,22)=5.15, p<0.05)$. Similarly, the scores of "Strong in one-to-one defensive play" and "Do not lose in interpersonal play” related to physical strength aspects were significantly higher among the high ranking group than the low ranking group $(F(1,22)=4.11, p<0.05),(F(1,22)=4.41, p<0.05)$. Furthermore, the score for "Play with confidence all the time" related to mental strength aspects was significantly higher among the high ranking group compared to the medium ranking and low ranking groups $(F(1,22)=7.77, p<0.05)$.

\section{Discussion}

The objective of the present study was to elucidate the actual conditions of young players' competitiveness in 
Table 2. Overall score of four indices in the competition power evaluation of the players.

\begin{tabular}{cccccc}
\hline Index & $\begin{array}{c}\text { High rank } \\
\text { group } \\
(\mathrm{n}=8)\end{array}$ & $\begin{array}{c}\text { Medium rank } \\
\text { group } \\
(\mathrm{n}=8)\end{array}$ & $\begin{array}{c}\text { Low rank } \\
\text { group } \\
(\mathrm{n}=8)\end{array}$ & F value & Multiple comparison result \\
\hline Technical score & $17.00 \pm 3.93$ & $14.63 \pm 5.15$ & $11.63 \pm 4.44$ & n.s. & \\
Tactics score & $6.50 \pm 2.51$ & $5.63 \pm 2.26$ & $4.88 \pm 2.23$ & n.s. & \\
Physical strength score & $15.50 \pm 5.29$ & $11.88 \pm 2.64$ & $10.00 \pm 3.30$ & $4.09^{*}$ & High rank group > Low rank group \\
Mental strength score & $18.25 \pm 2.92$ & $16.25 \pm 3.99$ & $13.50 \pm 2.78$ & $4.25^{*}$ & High rank group > Low rank group \\
\hline${ }^{*} p<0.05$, n.s.: not significant. & & & & &
\end{tabular}

various countries where the Social Action Program in Asia has dispatched instructors. Based on the findings of the study, we planned to develop training content and improvements that would be vital for the development of football in these countries.

The present study investigated the following four items pertaining to competitiveness: physical strength aspects, technical aspects, tactical aspects, and mental strength aspects. These four aspects are necessary factors in football (Kato et al., 1994). Continuous development by taking the optimal balance between the training burden and rest (Gero Bisanz et al., 1997) into consideration is essential for improving these four elements. In terms of training, these four items are related in the order of physical strength, techniques, tactics, and mental strength aspects. If there is a lack of continuous physical training, players will feel fatigue and this will affect their technique, such as passing and shooting accuracy. Subsequently, due to the fatigue, players would become unable to focus on precise movements and to think, which will greatly affect their tactical judgment. Tudor O. Bompa (2006) suggested that mental factors are dependent on the improvement of physical ability, which leads to further confidence and mental strength. This implies that perfect physical strength leads to the best state of mind.

In the present study, "physical strength score" was significantly higher among the high ranking group than the low ranking group. This is presumably due to the effect of the coaching systems of the high ranking countries. Mr. Takuma Koga is currently the head coach of the National Football Academy U-17 Team in Singapore, which is in the high ranking group. According to Mr. Koga, there are football teams for children U-13 to U-18 on a school wide basis and there are affiliated organizations of professional football club teams in addition to the grassroots football for children U-8 to U-12 (JFA, 2012a). Mr. Yoshinobu Yamaguchi served as a coach at the Bangladesh Krira Shikkha Protishtan (BKSP, Bangladesh Institute of Sports). This institution was established in 1986 as the national sports institute of Bangladesh. He stated that residential schools were established in five locations in all districts of Bangladesh and these schools successfully send 3 to 10 players to the U-14 to U-22 national football teams. There are even players who play in the national pro league (JFA, 2012b). On the other hand, Mr. Koji Gyotoku who served as the head coach of the Bhutan national football team, which is in the low ranking group commented that for further development, better football grounds and continuous and planned training for young players are required. Moreover, many games with foreign teams are necessary to promote development. He also pointed out the need for youth and coach development (JFA, 2009a). According to Mr. Kokichi Kimura who serves as a head coach of the Laos national football team stated that there are no football club activities, or teams on a school wide basis. Also, there are only a few children who play football in affiliate organizations of the Laos football league. He made the remark that there is an immediate need to establish football clubs for young people, educate corresponding instructors to nurture young people, and develop a positive environment to foster the game (JFA, 2014). These reports indicate that high ranking countries have football teams on a school wide basis and are affiliated with professional club teams. We speculate that high ranking countries have a significantly higher "physical strength score" compared to low ranking countries, because of the better environment. Such an environment allows continuous development while taking into account an optimal balance between the training burden and rest. Accordingly, it is suggested that the low ranking group needs to immediately improve their environment by establishing football teams on a school wide basis and get affiliated with organizations of professional club teams for each youth category. Moreover, there is also an urgent need to enhance physical strength by implementing continuous training.

The high ranking group compared to the low ranking group also had a significantly higher "mental strength score." According to Mr. Norio Tsukitate who serves as the head coach of the Guam national football team, which is in the low ranking group, training in Guam was less prioritized when he first arrived. However, as a result of continuous training effort, the "football mindset" was engrained in the players. They began to prioritize 
Table 3. Score of all items in the competition power evaluation of the players.

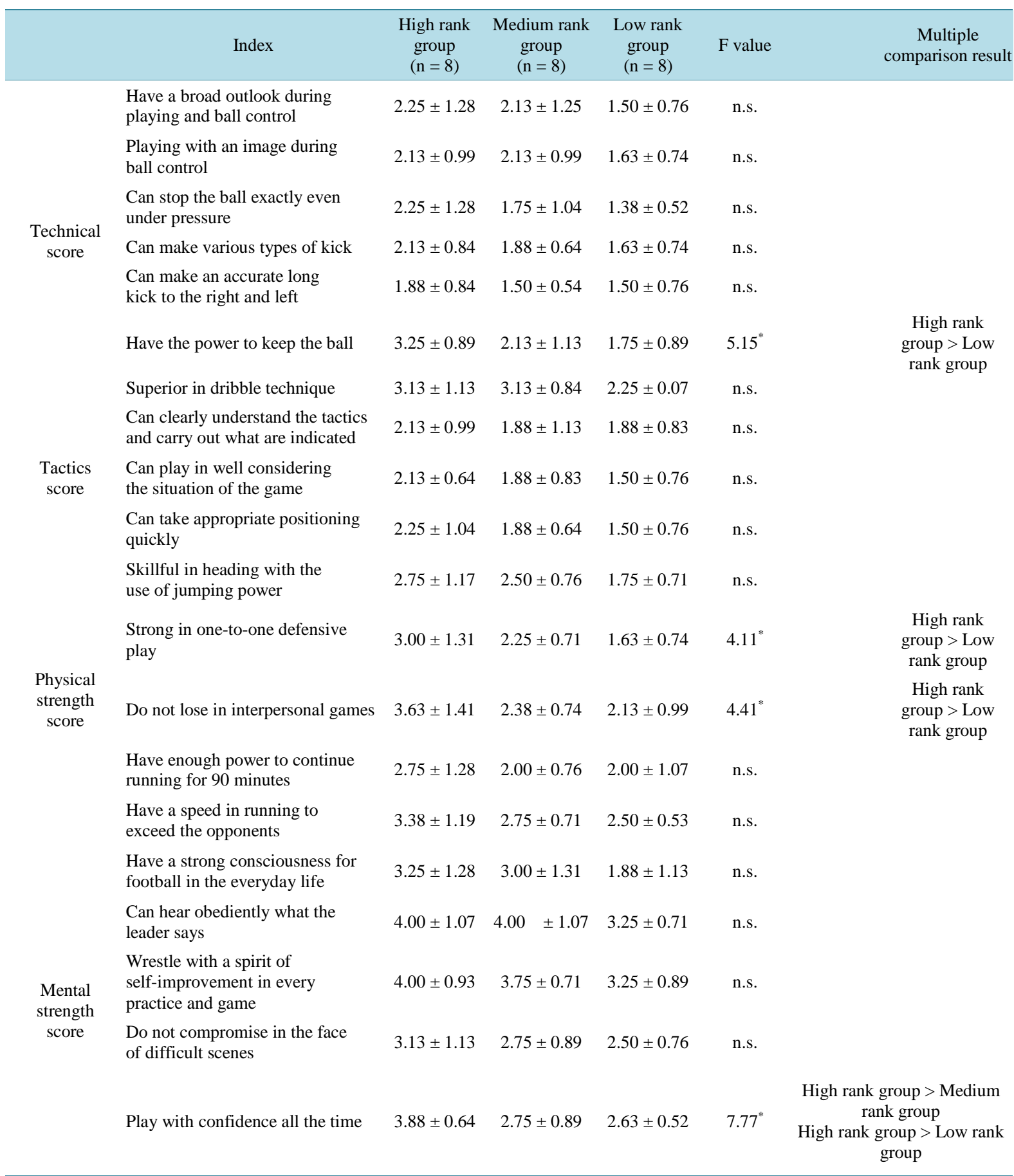

${ }^{*} p<0.05$, n.s.: not significant.

training more and eventually gained a sense of pride in representing their country by playing football (JFA, 2009b). Similarly, Mr. Masanaga Kageyama who serves as a head coach of the Macau national football team indicated that football is not the first priority in daily life of players in Macau, because, it is necessary to achieve a comprehensive balance between pride, meaning of becoming a representative player, the a sense of improvement and fulfillment from participating in training and games, in order to stimulate the motivation of players (JFA, 2007a). As indicated by the results of this study, the high ranking group had a significantly higher "mental 
strength score" compared to the low ranking group. We speculate that this was because players in the low ranking countries were less likely to prioritize football in their daily life and did not engage in continuous training. It is suggested that the low ranking countries should prioritize their training more. They should also cultivate a sense of pride in representing their country by playing football, as a result of achieving a comprehensive balance between the sense of improvement and fulfillment from participating in continuous training and games.

In terms of each factor score of the 20 items on the Players' Competitiveness Evaluation Scale, the score of "Have the power to keep the ball” related to technical aspects was significantly higher among the high ranking group than the low ranking group. Similarly, the scores of "Strong in one-to-one defensive play" and "Do not lose in interpersonal play" related to physical strength aspects were significantly higher among the high ranking group than the low ranking group. Additionally, the score of "Play with confidence all the time" related to mental strength aspects was significantly higher among the high ranking group compared to the low ranking group.

Matsuyama who is the national coach of Bhutan, which is in the low ranking group, pointed out the following regarding technical aspects (Matsuyama et al., 2014), "Have the power to keep the ball” He stated that there was a need to fully focus on basic training due to the lack of techniques and tactics among young players. Mr. Kazuo Kuroda is currently the head coach of the Chinese Taipei national U-18 football team, which is a medium ranking group. He also commented that the skill levels of Chinese Taipei players are lower compared to Japanese players of the same generation and that their basic passing skills are not thoroughly developed (JFA, 2013b). The above reports and the results of the present study suggest that basic skills and techniques are not fully developed among young players in the low and medium ranking groups, compared to the high ranking group. According to Ono (2010), basic skills and techniques such as ball control, passing, and shooting are requisite to compete with the world. He also mentioned that accumulation of training starting from a young age was essential. We speculate that there is a need to have a long-term vision, start basic training from a young age, and thoroughly develop and implement programs for the players. This will equip players with basic skills and techniques and help the low and medium ranking groups to bridge the gap with the high ranking group.

Similarly, Matsuyama et al. (2014) who is the national coach of Bhutan, which is in the low ranking group, have stated the following regarding physical strength aspects, such as "Strong in one-to-one defensive play" and "Do not lose in interpersonal play." He commented that there are many players who rarely train. As a result, they are not fully prepared for international games in terms of physical strength, due to a lack of practical physical training such as one-on-one defense, and contact play that are necessary for games. According to Mr. Kiyoshi Sekiguchi who is the head coach of the Northern Mariana Islands national football team and Mr. Kazunori Ohara who serves as the head coach of the Bhutan national football team, they focus on practical training using a ball while incorporating elements of physical fitness due to a lack of continuous development and nurturing of players in different categories (JFA, 2011, 2013c). The above reports and the results of the present study suggest that physical fitness is less well developed among young people in the low ranking group compared to the high ranking group. Wakamatsu (2013) stated that it is critical to go beyond physical training and incorporate physical elements in technical and tactical training while taking into consideration the fundamentals of football and its unique characteristics. Similarly, Bangsbo et al. (2008) advocated that physical training without a ball is neither professional, nor effective as football training. He stated that the best training methods need to improve technical and tactical skills, in addition to physical strength, and doing so under highly motivated conditions would enhance physical strength though ball training and actual games. Therefore, it is necessary to develop physical strength by incorporating ball training and training that are based on the fundamentals of football and its unique characteristics. This will help the low ranking group to bridge the gap with the high ranking group.

Matsuyama who is the head of the Bhutan national football U-19 team reported on mental strength aspects such as, "Play with confidence all the time," and stated that it was difficult for players to play confidently, because they rarely had regular, routine training, or international game experiences (Matsuyama et al., in press). The above report and the results from the present study suggest that mental training is limited among the low ranking group compared to the high ranking group due to insufficient game experience including official games. Takahata (2006) stated that it is important for players to have a satisfying amount of training so that they could feel they have done all they could for the day. He also acknowledged the importance of the accumulation of successful experiences. Based on the above, we suggest that it is necessary to continue training, increase opportunities to experience games including international games, and accumulate successful experiences. This will help the low and medium ranking groups bridge the gap with the high ranking group. 


\section{Conclusion}

There are limited technical and tactical training available for the low ranking group in the FIFA rankings. As a result, there is a decline in physical fitness among the countries where instructors have been dispatched as a part of the Social Action Program in Asia. For this reason, players in low ranking countries have difficulty to play with confidence during regular routine training, or in international competitions. Therefore, it is suggested that the low ranking group needs to have a long-term vision and exclusively focus on continuous development of players from a young age, according to each youth category. It is also crucial to improve the environment to promote youth development in order to increase their competitiveness.

One method to improve the environment for players by promoting youth development is to organize each category of U-12, U-15, and U-18 by age, similar to JFA (JFA, 2007b). Also, there is a need to improve the environment for the players so that talented players can participate in training and games on a regular basis (Bert van Lingen, 2003). Nishi (2008) also commented that leading football powerhouses attach great importance to young people, and development from a young age can facilitate further growth. This suggests that low ranking countries need to immediately improve the environment for players, in order to promote youth development.

\section{Summary}

Conditions affecting the competitiveness of young group athletes in different countries were investigated through a comparative evaluation of opinions expressed by coaches and instructors dispatched overseas through the JFA Social Action Program in Asia. Results of a one-way ANOVA on responses scores related to football teams in different countries, which was conducted after calculating group means and standard deviations indicated that physical strength and mental strength scores were significantly higher in the high rank group than in the low rank group classified according to FIFA rankings. Results of factor scores indicated that the score for "Have the power to keep the ball" which is a technical skill, was significantly higher among the high ranking group compared to the low ranking group. Similarly, the scores for "Strong in one-to-one defensive play" and "Do not lose in interpersonal play," related to physical strength aspects was significantly higher among the high ranking group compared to the low ranking group. Additionally, the score for "Play with confidence all the time," which is a mental strength aspect was significantly higher in the high ranking than in the low and medium ranking groups. These findings indicated significant differences between the contents of technical aspects, physical strength aspects, and mental strength aspects between players in the high and lower ranking groups. These results suggest an urgent need to establish football teams on a school wide basis, and to become affiliated organizations of professional football clubs, in order to implement continuous training for each category of young athletes. Such a program is necessary to increase the physical strength and thereby decrease differences in physical strength scores between high and low ranking groups. Similarly, to bridge the gap in mental strength scores between low and high rank groups, it would be important that young athletes continue training, participate in games, and feel a sense of improvement and fulfillment. A comprehensive balance between training, playing games, and obtaining a sense of fulfillment is expected to lead to prioritized training and develop a sense of pride among young athletes representing their country by playing football. It is suggested that training programs be developed to: 1) Enhance the level of basic techniques such as "Have the power to keep the ball," and maintain a long-term vision and thoroughly develop basic skills and techniques of players from a young age; 2) Improve the physical condition of players by developing "Strong in one-to-one defensive play" and "Do not lose in interpersonal play." Moreover, it is suggested that continuous training is essential to increase and maintain physical strength. Furthermore, young players need training based on fundamentals of football and its unique characteristics including ball training; 3) Strengthen mental strength aspects such as "Play with confidence all the time." It is also vital that young players continue training by focusing on the quantity and content of accumulate successful experiences to build their confidence.

\section{References}

Abe, H., \& Ochiai, M. (2012). The Relationship between Soccer Coach’s Expectancy and Coaching Behavior. Journal of Educational Research, 26, 55-67.

Bangsbo, J., Hasegawa, H., Yasumatsu, M., \& Ueda, J. (2008). Aerobic and Anaerobic Training in Soccer (pp. 8-54). Tokyo: Taishukan, Co., Ltd. 
Bert, V. L. (2003). Cochen Van jeugdvoet Ballers (pp. 204-205). Tokyo: Taisyukan, Co., Ltd.

Federation Internationale de Football Association (2014). FIFA/Coca-Cola World Lanking. http://www.fifa.com/fifa-world-ranking/index.html

Gero, B., Gunnar, G., \& Tajima, K. (1997). Rowohlt Taschenbuch Verlag GmbH (pp. 27-47). Baseball Magazine, Co., Ltd.

International Olympic Committee (2014). Sports Leader Foreign Countries Training 2013 Returnee Report (pp. 55-87). Tokyo: Japanese Olympic Committee.

Japan Football Association (2007a). Technical News (Vol. 19, pp. 40-41). Gifu: Sun Messe, Co., Ltd.

Japan Football Association (2007b). Guidelines about the Game Environment of the Upbringing Generation (pp. 2-7). Tokyo: Asahi Business, Co., Ltd.

Japan Football Association (2009a). Technical News (Vol. 31, pp. 42-43). Gifu: Sun Messe, Co., Ltd.

Japan Football Association (2009b). Technical News (Vol. 34, p. 45). Gifu: Sun Messe, Co., Ltd.

Japan Football Association (2010). U-12 Instruction Guidance 2010 (pp. 4-6). Tokyo: Asahi Business, Co., Ltd.

Japan Football Association (2011). Technical News (Vol. 44, pp. 46-47). Gifu: Sun Messe, Co., Ltd.

Japan Football Association (2012a). Technical News (Vol. 49, pp. 42-43). Gifu: Sun Messe, Co., Ltd.

Japan Football Association (2012b). Technical News (Vol. 51, pp. 42-43). Gifu: Sun Messe, Co., Ltd.

Japan Football Association (2013a). JFA PLOFILE (pp. 1-26). Gifu: Sun Messe, Co., Ltd.

Japan Football Association (2013b). Technical News (Vol. 58, p. 43). Gifu: Sun Messe, Co., Ltd.

Japan Football Association (2013c). Technical News (Vol. 56, pp. 38-39). Gifu: Sun Messe, Co., Ltd.

Japan Football Association (2014). JFA Dream Asia Project.

http://www.jfa.jp/social_action_programme/international_exchange/dispatch.html

Japan Football Association (2014). Technical News (Vol. 60, pp. 42-43). Gifu: Sun Messe, Co., Ltd.

Kato, K., Hukubayashi, T., Omori, K., Yamamoto, T., Yano, M., Nishijima, N., Oyama, M., \& Yoshida, Y. (1994). Soccer Training Bible (pp. 130-142). Tokyo: Dainihon, Co., Ltd.

Kobayashi, T. (2001). A Study on the Impact of the Expansion of Global Sport on Developing Countries. The Case of the Melanesia Region. Japan Journal of Sport Sociology, 9, 83-93. http://dx.doi.org/10.5987/jjsss.9.83

Matsuyama, H., \& Tsuchiya, H. (in Press). The Current Situation and Challenges of Sports Instructors Overseas on Dispatching Project-Based on Its Activities of Strengthening Bhutan National U-19 Football Team. Journal of Japan Society of Sports Industry, 25, 1.

Matsuyama, H., Tsuchiya, H., Horino, H., \& Suda, Y. (2014). The Current Situation and the Problems of the Sport Coach Dispatch Business-Thorough Coaching Bhutanese National Football Team in JFA DREAM ASIA PROJECT. Journal of Osaka Society of Physical Education, 52, 15-22.

Nishi, M. (2008). Importance and a Problem of the Upbringing Period Consistency Guidance in the Japanese Soccer: Player Upbringing to Work in the World. The Kyotogakuen University Review, 18, 173-196.

Ono, T. (2010). Football Player Report (pp. 226-230). Tokyo: Kanzen Co., Ltd.

Sone, J. (2008). A Study on the Ways to Make the Strategy and Tactics in SYRIA U-17 National Football Team. Journal of Osaka Society of Physical Education, 39, 37-38.

Tudor, O. B., Ogata, M., \& Aoyama, K. (2006). Theory and Methodology of Training (4th ed., pp. 38-55). Tokyo: Taishukan, Co., Ltd.

Wakamatsu, K. (2013). The Basic Method of Sports Training (pp. 184-193). Chiba: Seitosha, Co., Ltd. 